\title{
Design and validation testing of a dynamic ankle orthosis
}

\begin{abstract}
A new Dynamic Ankle Orthosis (DAO) was designed to provide the coupled benefits of ankle offloading while supporting the functional range of motion of the natural ankle joint to non- invasively alleviate pain due to ankle osteoarthritis without increasing the muscle effort to move the ankle. The DAO used pneumatic cylinders to apply a controlled distractive force across the ankle joint and tie rod ball joints to allow functional ankle motion. The objectives of this study were to confirm the brace offloading effect during static double stance and quantify the muscle effort needed to move the ankle through a functional range of dorsiflexion and plantar flexion. The static offloading effects of the DAO were measured at varying levels of cylinder pressure using load cells. The ankle passively moved through the functional ROM of $10^{\circ}$ dorsiflexion, and $20^{\circ}$ plantar flexion in a non-weight bearing setting using a Biodex Isokinetic Dynamometer with and without the brace donned. This was also done at varying levels of cylinder pressure. The DAO prototype was able to offload between $11 \%-30 \% \mathrm{BW}$ and transferred cylinder forces to the lower limb at rates between $77 \%-95 \%$. The DAO allowed for full functional ROM of the ankle. No significant difference was found in resistive ankle moment between the no brace and deactivated brace configurations. With increasing cylinder pressure, there was a significant reduction in baseline resistive moment at the ankle. The amount of resistive moment difference represented $2 \%$ of the maximal ankle moment experienced during walking.
\end{abstract}

Keywords: foot ankle orthosis, mechanical design, brace
Volume 2 Issue 3 - 2018

\author{
Chloe L Chung and Denis J DiAngelo \\ Department of Orthopedic Surgery and Biomedical Engineering. \\ University of Tennessee Health Science Center, USA
}

Correspondence: Denis J DiAngelo, Department of Orthopaedic Surgery and Biomedical Engineering The University of Tennessee Health Science Center 956 Court Ave, Suite E226 Memphis,TN 38163, USA, Email ddiangelo@uthsc.edu

Received: May 25, 2018 | Published: June 07, 2018
Abbreviations: DAO, dynamic ankle orthosis; OA, osteoarthritis; PTOA, Post-traumatic osteoarthritis; AFO, ankle foot orthosis; ROM, range of motion

\section{Introduction}

Ankle fractures represent a significant portion of orthopedic trauma injuries that occur in the United States. In 2014, over 60,000 workrelated ankle injuries were reported to the U.S. Department of Labor ${ }^{1}$ with average costs up to $\$ 5,900$ per injury. ${ }^{2}$ Patients who sustain irreversible cartilage damage or joint instability from ankle injuries are likely to develop ankle arthritis. Affecting approximately $1 \%$ of adults, ${ }^{3}$ ankle osteoarthritis (OA) is a degenerative disease that occurs when the cartilage layer in the joint wears away over time. Unlike OA of other joints, $70 \%-80 \%$ of ankle OA cases are associated with a previous traumatic injury, a condition known as the Post-Traumatic Ankle Osteoarthritis (PTOA)., ${ }^{3,4}$ Long-term physical impairments, joint pain, and reduced quality of life are all possible outcomes of this debilitating disease. Ankle OA creates progressive pain within the ankle joint, inflammation of the joint, and substantial decrease in overall range of motion and stability of the joint due to joint articular cartilage erosion. Invasive treatment options include ankle replacement (arthroplasty) and ankle fusion (arthrodesis). There are approximately 80,000 ankle fusions and 16,000 ankle replacements performed annually, ${ }^{5}$ and the estimated economic burden of foot and ankle surgery in the Medicare population was \$11 billion in 2011. However, surgical procedures are joint sacrificing and typically done as a last resort. The pain and inflammation associated with PTOA can be managed noninvasively using modified footwear and orthotics such as an Ankle-Foot Orthosis (AFO) (i.e., brace).
Traditional AFOs can have solid joints with locked ankle position to prevent uncontrolled plantar flexion (i.e., foot drop due to weak dorsiflexors) or toe drag during walking. These solid AFOs are often comprised of lightweight plastic material that has been custom molded to fit the patient and maintain a desired ankle position. AFOs can also be designed with articulating pin joints to provide either free or controlled sagittal plane ankle motion. By incorporating a feature that provides a physical plantar flexion stop, plantar flexion resistance, or dorsiflexion assistance, the brace can prevent excessive plantar flexion while permitting natural progression of the tibia over the foot and natural dorsiflexion of the foot during the stance phase of walking. Articulating AFOs can be made with plastic materials or using metal and leather components adapted to the user's existing footwear. Ultimately, the biomechanical function and design of the prescribed AFO must match the needs of the patient. ${ }^{7}$ A standard double upright AFO (DUAFO) is commonly prescribed to treat PTOA symptoms. These braces provide stabilization of the ankle by limiting ankle walking motion (i.e., sagittal plane). ${ }^{8}$ However a standard DUAFO (Figure 1) has important limitations in that it functions primarily to stabilize the ankle joint with restricted motion and provides minimal to no off-loading. Reduced axial loads through the ankle have been shown to be effective in reducing the pain and discomfort of ankle arthritis. ${ }^{9}$ A device that preserves ankle mobility while offloading the ankle joint for alleviating the pain of PTOA is not currently available on the market. A new Dynamic Ankle Orthosis (DAO) was designed to provide the coupled benefits of ankle offloading while supporting the functional range of motion (ROM) of the natural ankle joint to noninvasively alleviate pain due to ankle osteoarthritis without increasing the muscle effort to move the ankle. The objectives of this study were to confirm the brace offloading effect during static double stance 
and quantify the muscle effort needed to move the ankle through a functional range of motion in the sagittal plane (i.e., dorsiflexion and plantar flexion).

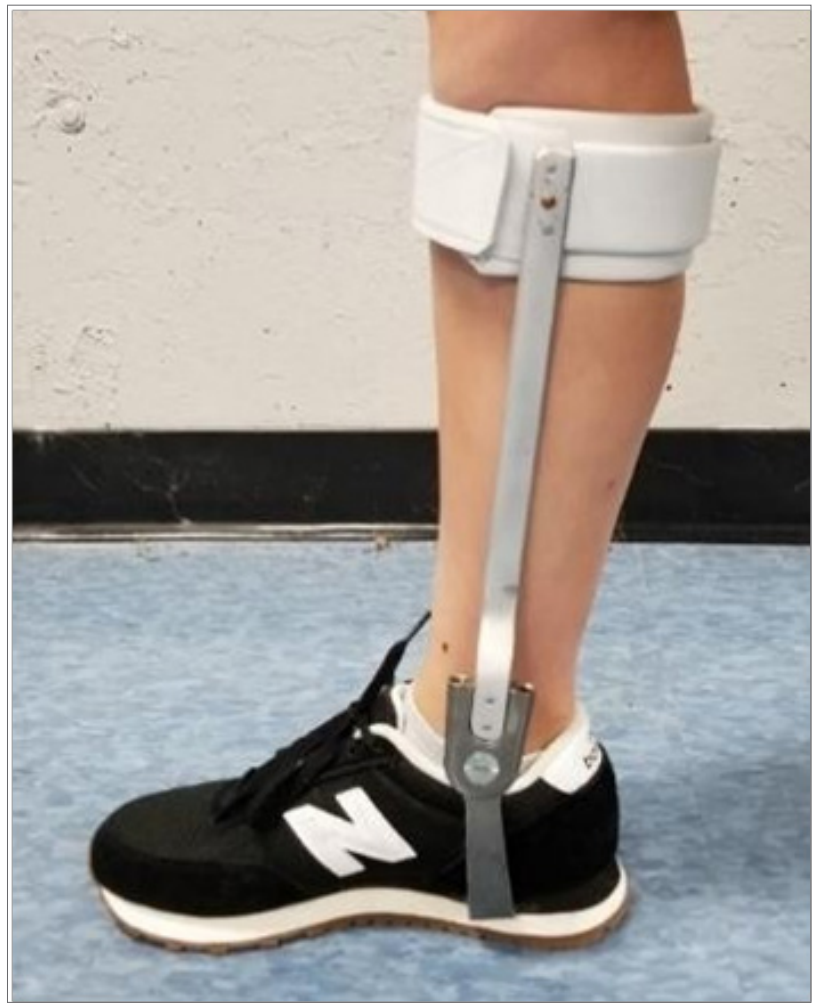

Figure I The standard of care leather and metal double upright ankle-foot orthosis.

\section{Methods}

The design goals of the DAO device were two-fold: 1) offload the ankle joint by applying a distractive force between the foot and proximal tibia and 2) allow for a functional range of motion at the natural ankle joint without increasing the muscle effort required to move the ankle. The DAO (Figure 2) consisted of three main components: a calf sleeve, a foot plate, and two pneumatic cylinders that attached to the calf sleeve and foot plate via ball joints and tie rod ends and served as a distractive force mechanism (DFM). The DAO applied a distractive force along the axis of the lower leg and through the rotational axis of the device to create an offloading effect. The two cylinders were connected with tubing so that air could move between cylinders as needed (i.e., when one cylinder rod collapsed, the other cylinder rod extended). This action allowed the actuator force to remain constant during weight bearing as the foot moved into inversion and eversion. The ball joints and tie rod ends, coupled with the way the actuators were configured, allowed for functional ranges of inversion, eversion, dorsiflexion, and plantar flexion of the ankle joint. Based on recommendations of local Orthotists (Center of Prosthetics and Orthotics Inc.) the brace was designed to offload at least $10 \%$ of the user's body weight (BW). Finally, the arrangement of the device's rotational axis with the anatomical axis of the ankle joint allowed for a functional walking range of dorsiflexion $\left(\sim 10^{\circ}\right)$ and plantar flexion $\left(\sim 20^{\circ}\right)$ (i.e., rotating toes up and down) (Figure 3 ), and some amount of inversion and eversion (i.e., side-to-side rotation) (Figure 4). To test the effectiveness of the DAO to meet the design goals, two experiments were performed: a first experiment was conducted to confirm how much offloading the DAO provided at the lower limb, and a second experiment was conducted to test the changes in resistance to ankle plantar flexion and dorsiflexion compared to the native ankle condition. The methodology of these experiments is provided below.
(A)

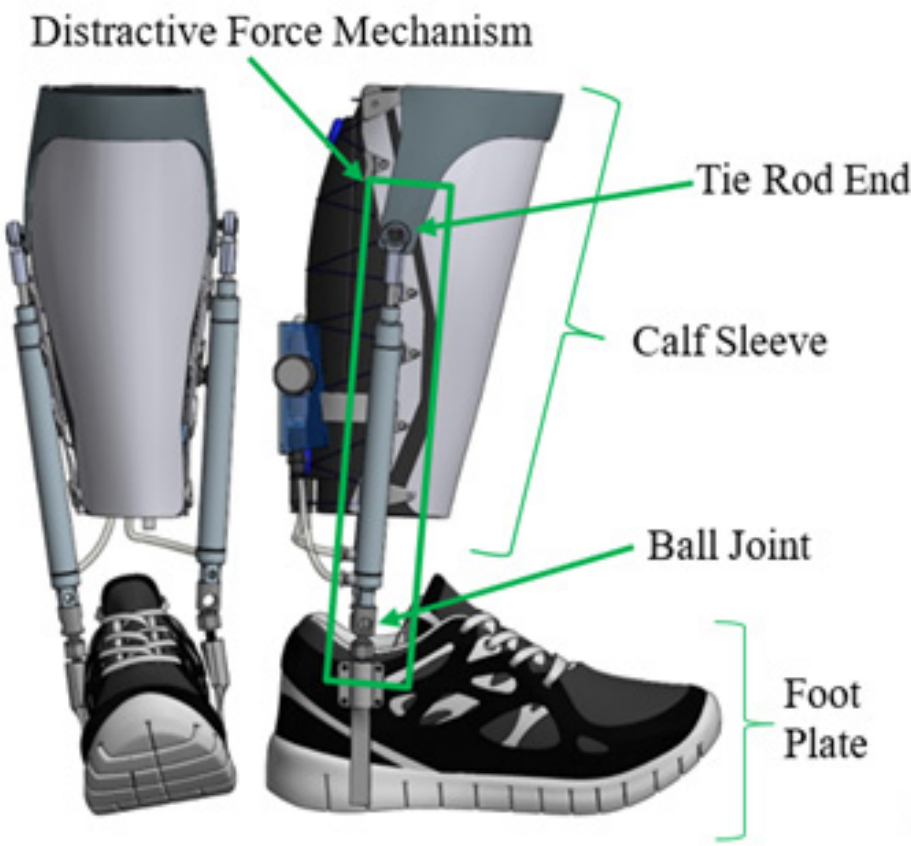

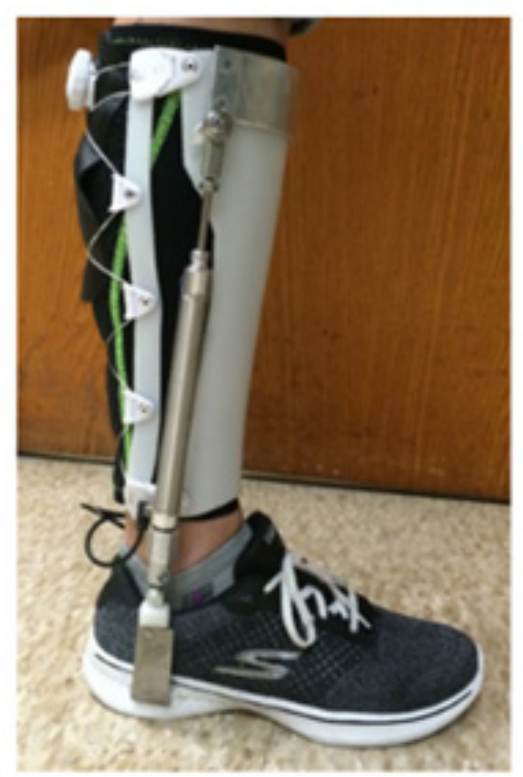

Figure 2 Dynamic Ankle Orthosis (A) concept model with components and (B) prototype. 
(A)

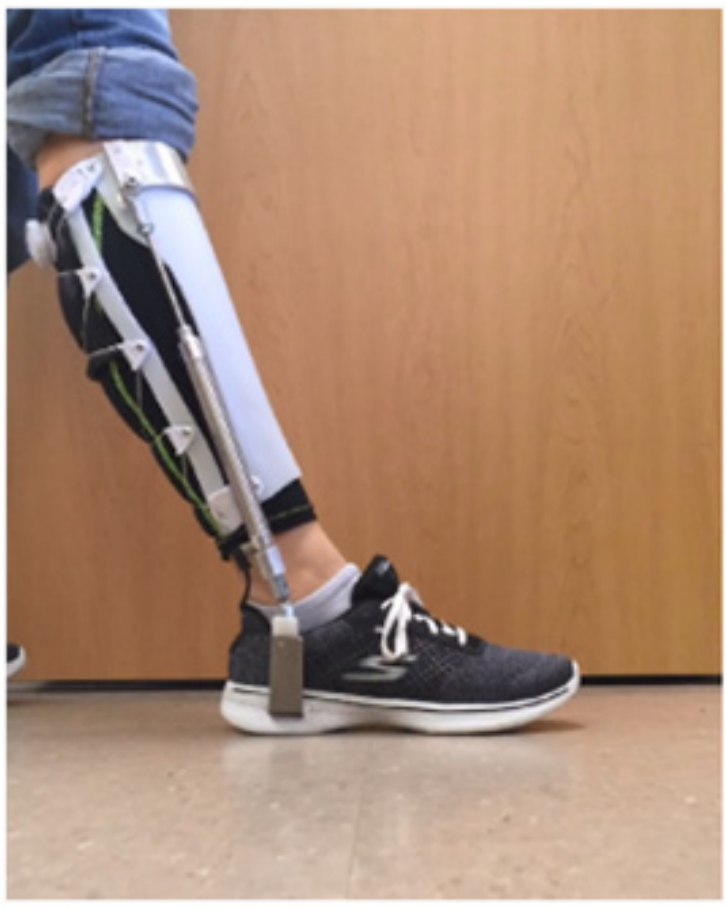

(B)

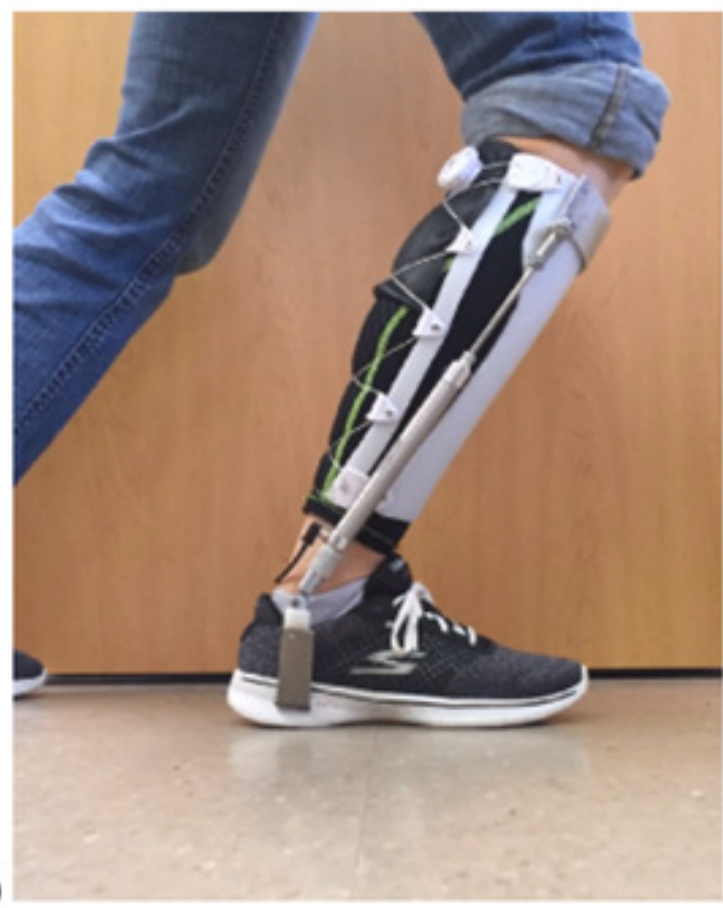

Figure 3 The sagittal plane range of motion of DAO at (A) maximum plantar flexion and (B) maximum dorsiflexion in weight bearing.

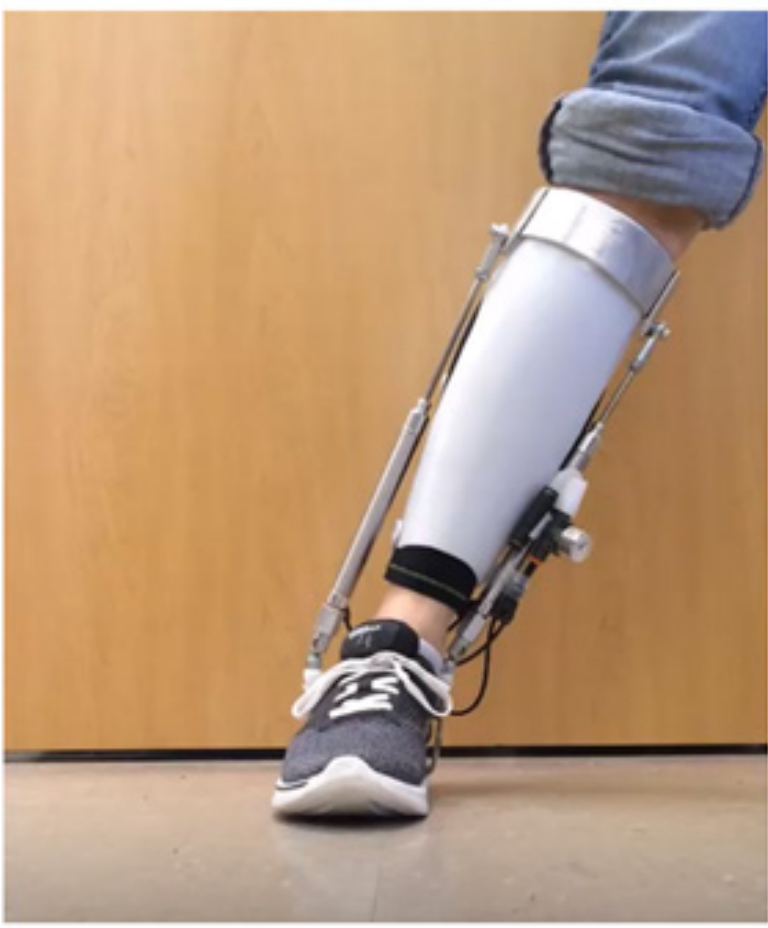

(A) (B)

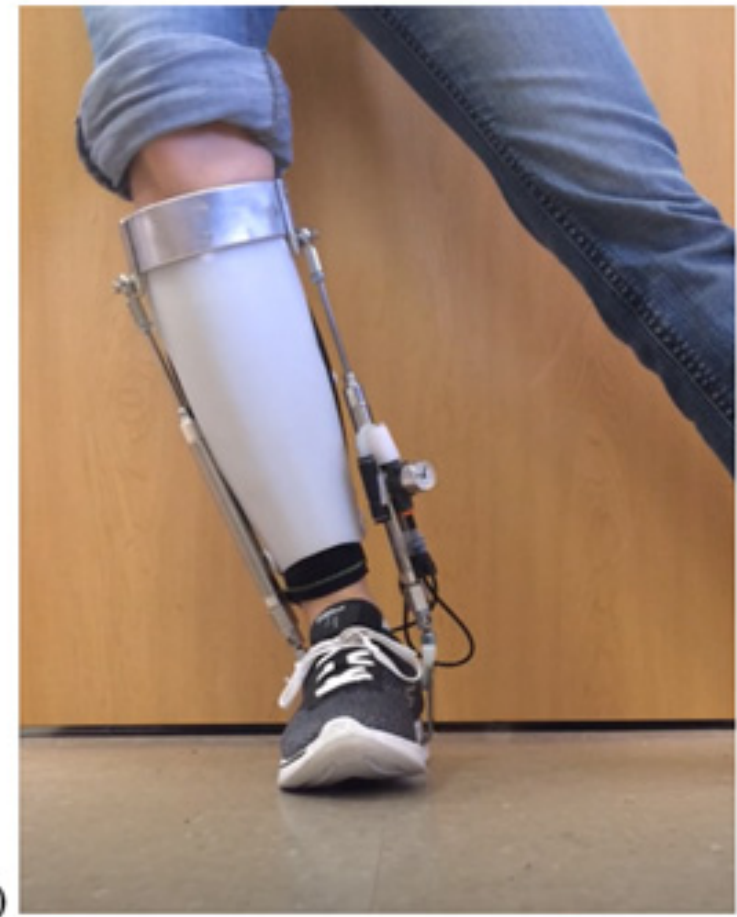

Figure 4 The frontal plane range of motion of DAO at (A) maximum inversion and (B) maximum eversion in weight bearing. 


\section{Offloading validation}

This experiment was performed in two parts: 1) define cylinder pressure-output force relationship of the two cylinders alone, and 2) define the cylinder pressure-offloading relationship of the brace. The pressure-output force relationship of the stand-alone pneumatic cylinders was first characterized (Figure 5). A plate was created with two clearance holes to place the 7/16-20 threaded ends of the cylinders through. A corresponding nut was then used to secure pneumatic cylinders to the plate. They were each attached to a vertical fixture for positioning, which was securely attached to the platform of a robotic test frame and placed beneath a load cell (Model 100M40 six-axis load cell; JR3 Inc., Woodland, CA). The cylinders were connected in series in the same manner as when attached to the DAO. By connecting pneumatic cylinders in series, equivalent pressures were delivered to the two cylinders, corresponding to the pressure value shown on the pressure gauge (i.e., when the pressure gauge showed 40 PSI both cylinders were inflated to a pressure of 40 PSI). Both cylinders acted on the load cell, so the reported values show the total amount of force delivered by both cylinders. The compressive force on the load cell was acquired at various cylinder pressure values: 40, 50, 60, 70, and 80 PSI. Each targeted pressure value was tested four times, and the load cell readings were exported and processed in Microsoft Excel (2013). The pneumatic cylinders were emptied of their pressures between all runs and inflated to the targeted pressure to begin each new test.

To quantify the biomechanical offloading capabilities of the DAO as a function of pneumatic pressure relative to body weight, a testing fixture was created to measure the amount of load relief that the DAO provided (Figure 6). Two vertical fixture plates were mounted to a base plate and positioned on either side of the braced leg. A small mounting plate was attached to the vertical fixture plates, and the lower ends of the DFM pneumatic cylinders were attached to the mounting plate via tie rod ball joint connectors. The mounting plates were adjusted so that the height and location of the connectors were comparable to that of the two ankle malleoli. Two load cells (Model 100M40 and Model 67M25S3; JR3 Inc., Woodland, CA) were bolted to the top of the base plate, and wooden planks were then attached to the top of each of the load cells to give the user somewhere to stand. The user stood with their feet approximately shoulder width apart with one foot on one load cell and the other foot on the other load cell. With the lower end of the cylinders attached to the ball joint connectors and side mounting plates, the subject donned the calf sleeve of the DAO around their right leg. In this configuration, any brace force introduced by pneumatic cylinders bypassed the load cell under the braced limb and transferred to the base plate.

Testing was performed in static double stance with each foot positioned on top of a single load cell. A custom program recorded load cell readings over a specified period. At the start of each test, approximately five seconds of load data were collected without brace activation. The pneumatic cylinders were activated to a specific pressure to create a bracing force, and testing was continued for twenty seconds. The pneumatic cylinders were inflated to five different pressures: 40, 50,60, 70, and 80 PSI and tested ten times at each level. Brace force $(\mathrm{Fb})$ was calculated for each run by taking the user's bodyweight (BW), or the sum of the readings of the left load cell force (FLLC) and of the right load cell force (FRLC) before brace activation and subtracting out the sum of the two load cell readings after brace activation. Brace Efficiency, or how well DAO transferred offloading forces to the limb, was calculated using the brace force $(\mathrm{Fb})$ of the DAO and total cylinder force provided by pneumatic cylinders in the first part of this experiment.

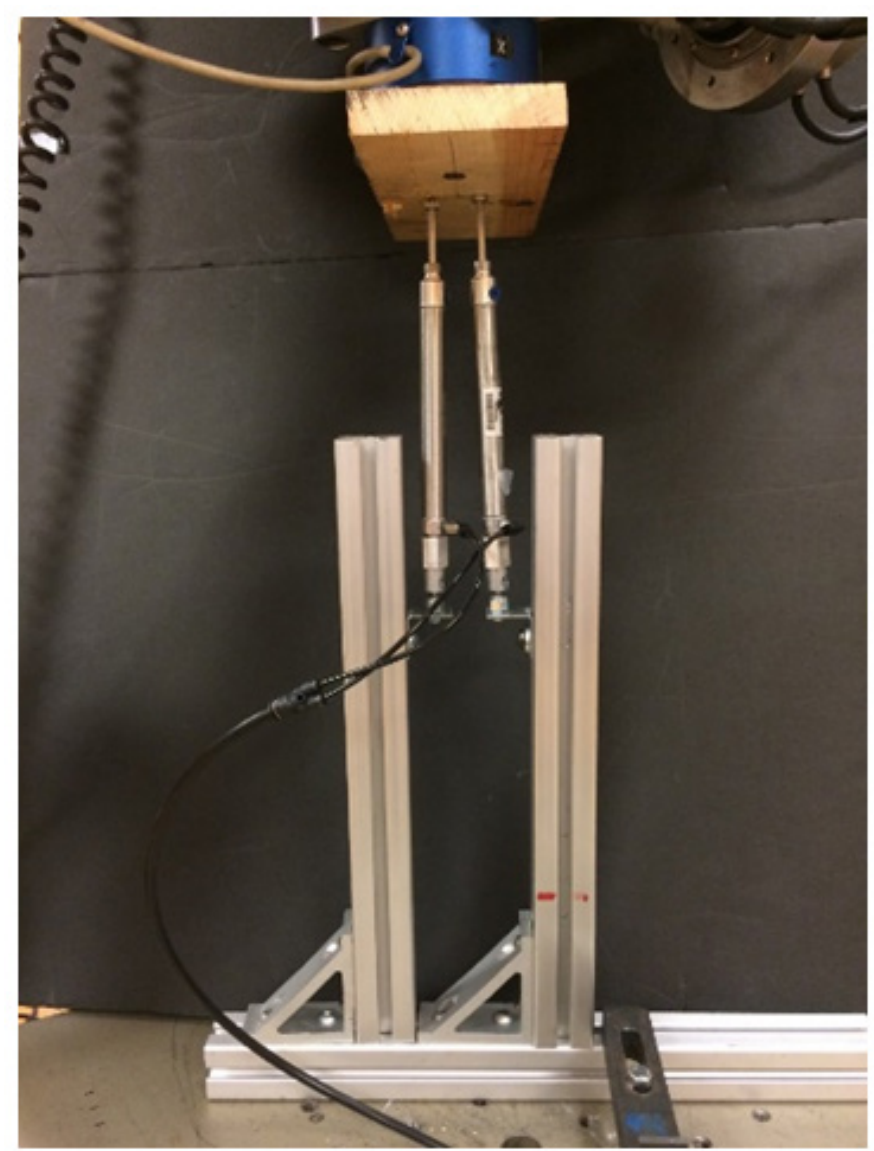

Figure $\mathbf{5}$ Setup for characterizing cylinder pressure-output force relationship.

\section{Mobility validation}

Validation of the second design goal of the DAO, i.e., to provide a functional range of ankle motion without increasing the muscle effort required to move the ankle joint, was done by analyzing the effect of the DAO on the ankle's resistance to passive sagittal plane motion. A Biodex Dynomometer (System 4 Pro Model: 850-000, Biodex Medical Systems, NY, USA) was set to passively drive the ankle in plantar flexion and dorsiflexion with and without the DAO worn. The user sat in a chair, and a limb support pad was placed under the thigh so that the lower leg approached the machine parallel to the floor (Figure 7). The right foot was placed on a foot plate and securely attached to limit motion in the foot. The footplate was then adjusted so that the dynamometer of the Biodex unit was aligned with the lateral malleolus of the right ankle. This was done so that the Biodex unit rotated about the rotational axis (RA) of the user's ankle. The Biodex unit was set to rotate the ankle between two set points at a set speed. The two points were set before each run by the user. The foot was initially positioned at a neutral angle, perpendicular to the long axis of the leg, and then the user sets maximum plantar flexion and dorsiflexion angles. The machine rotated back and forth between 
these maximum angles for a set number of cycles and measured the moments experienced by the machine while moving the foot to these points. The data sets attained from the tests were the angular positions and the corresponding moment values recorded by the dynamometer. All tests were run at an angular velocity of 30 degrees $/ \mathrm{sec}$. For all tests, the ankle was rotated to at least 10 degrees of dorsiflexion and 20 degrees of plantar flexion. The polarity of the measured moment values depends on the direction of the resistive moment. Dorsiflexor moments (directed towards the top of the foot) were measured as negative resistive moments, and plantarflexor moments (directed towards the bottom of the foot) were measured as positive resistive moments). So as the foot passively moved into dorsiflexion, a resistive plantarflexor moment was generated by the soft tissue of the calf and ankle.
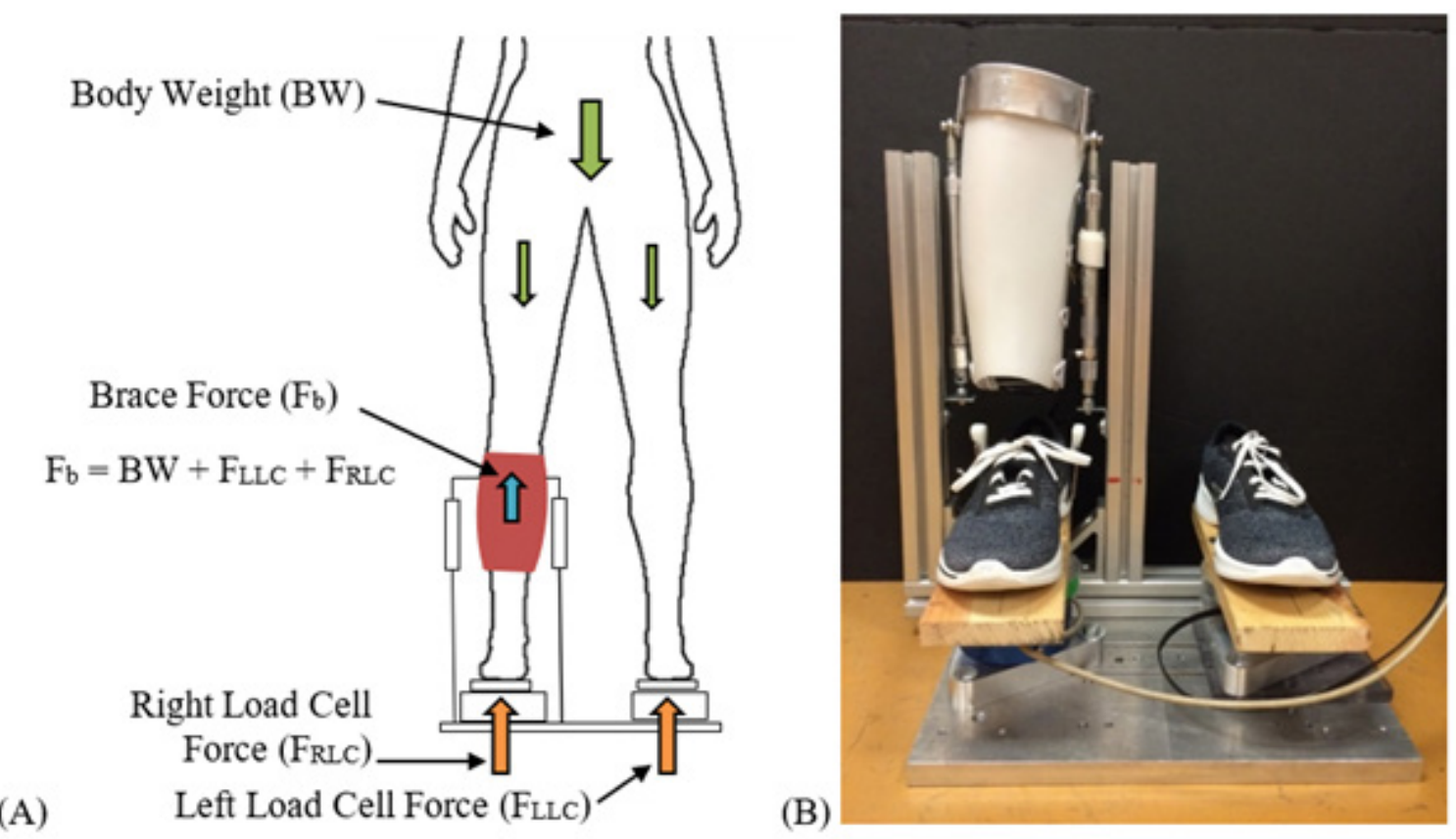

Figure 6. Setup for offloading validation (A) free body diagram and (B) test fixture.

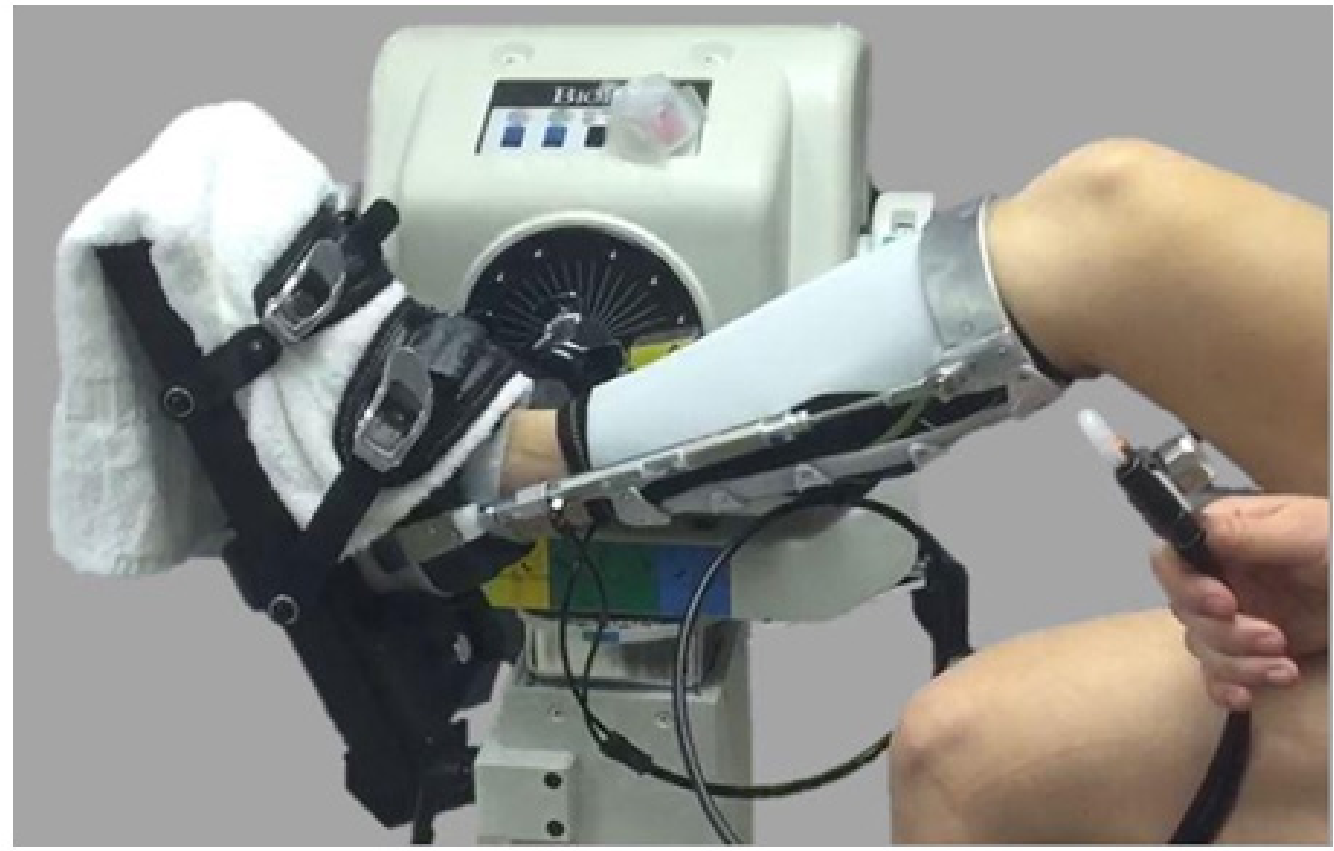

Figure 7 Setup for mobility validation of DAO using a Biodex Dynomometer. 
The foot was placed in the Biodex machine without the DAO donned. The straps were securely tightened at the foot to ensure that rotation occurred at the ankle joint. Three tests were run, each for thirty seconds with the system angular velocity set to $30 \mathrm{deg} / \mathrm{sec}$. The mean moments and standard deviations were then calculated at 5 degree increments from: 10 degrees to 20 degrees. Moment values for the plate by itself were subtracted out so the reported moment values accurately represent what was added to the Biodex system. These values provided a baseline to compare the results of the different bracing conditions to that of the foot alone. Five different bracing conditions were tested at the neutral position. The user sat in the chair as above, with the DAO donned and the foot secured to the footplate. Each test was run for 30 seconds and contained between 7 and 10 full revolutions of the foot, moving from maximum dorsiflexion to maximum plantar flexion and back. Tests were run with the pneumatic cylinders filled to $0,40,50,60,70$, and $80 \mathrm{PSI}$ values. Each pressure setting was run three times and the recorded output values were the moments and the corresponding angular position values.

Moment values at $-10,-5,0,5,10$, and 15 degrees were used for analysis. The mean resistive ankle moments were compared amongst all bracing conditions using a Shapiro-Wilk Normality Test, Equal Variance Test, One-Way ANOVA, and Holm-Sidak Post-Hoc Analysis (Sigma Plot Version 13.0; Systat Software Inc., CA). Significance was set at an alpha level of 0.05 . The null and alternative hypotheses were defined as follows: Ho: There was no difference in mean resistive ankle moment between bracing conditions; HA: There was a difference in mean resistive ankle moment between bracing conditions.

\section{Results}

\section{Offloading results}

The mean force values of the output force that the pneumatic cylinders exerted when filled to certain pressures are shown in Table 1 along with the mean transferred brace force, $\mathrm{Fb}$, that was computed from the load cell data per the free body diagram of Figure 6. Table 1 also shows the brace efficacy expressed as a percentage of the cylinder force that the brace was able to transfer in the form of a bracing force, $\mathrm{Fb}$. The percent bodyweight offloaded by the brace force $\mathrm{Fb}$, using the mean body weight of $490 \mathrm{~N}$ is shown in Figure 8 .

Table I Activated cylinder pressures with corresponding mean output force for two pneumatic cylinders, computed transferred brace force, and brace efficacy

\begin{tabular}{llll}
\hline Pressure[PSI] & $\begin{array}{l}\text { Cylinders output } \\
\text { force[N] }\end{array}$ & $\mathbf{F b}[\mathrm{N}]$ & $\begin{array}{l}\text { Brace } \\
\text { efficiency }\end{array}$ \\
\hline 40 & $65.5 \pm 2.5$ & $54.7 \pm 7.3$ & $83.40 \%$ \\
50 & $87.2 \pm 0.7$ & $75.1 \pm 3.7$ & $86.10 \%$ \\
60 & $111.9 \pm 2.2$ & $106.9 \pm 5.9$ & $95.40 \%$ \\
70 & $135.5 \pm 2.6$ & $123.5 \pm 5.9$ & $91.40 \%$ \\
80 & $155.3 \pm 1.3$ & $148.0 \pm 7.9$ & $95.50 \%$ \\
\hline
\end{tabular}

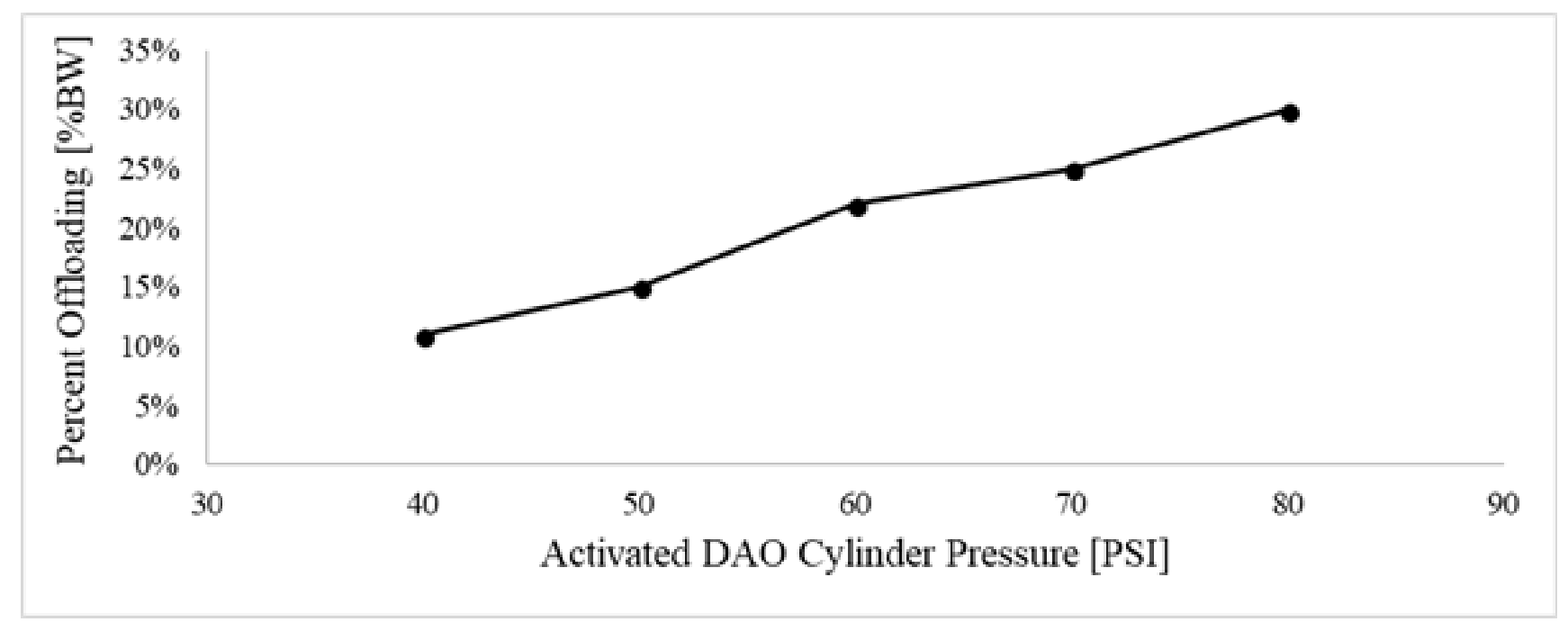

Figure 8 Percent of bodyweight offloaded by the DAO at varying levels of cylinder pressure.

\section{Mobility results}

Following in Table 2 are the mean moment values for the native ankle and braced conditions. At each five degrees increment in both directions mean moment was compared amongst all different bracing conditions. An angular ankle position of 0 degrees corresponds to the neutral ankle position where the foot makes a 90-degree angle with the long axis of the lower leg. Negative angle values indicate dorsiflexion and positive values plantar flexion. A box plot representation of the resistive ankle moments measured at each angular ankle position for each of the bracing conditions is shown in Figure 7. The mean resistive ankle moment for all DAO bracing conditions is significantly different from "Native Ankle" except for "DAO 0PSI" condition. 
Table 2 Native ankle and braced ankle moment values $[\mathrm{Nm}]$ at $5^{\circ}$ increments of angular ankle position

\begin{tabular}{llllllll}
\hline & \multicolumn{2}{c}{ Dorsiflexion (-) } & \multicolumn{4}{c}{ Neutral } & \multicolumn{4}{c}{ Plantar flexion (+) } \\
\cline { 2 - 7 } & $-\mathbf{1} \mathbf{0}^{\circ}$ & $\mathbf{- 5}^{\circ}$ & $\mathbf{0}^{\circ}$ & $\mathbf{5}^{\circ}$ & $\mathbf{1 0 ^ { \circ }}$ & $\mathbf{1 5 ^ { \circ }}$ & $\mathbf{2 0 ^ { \circ }}$ \\
\hline Native ankle & 6 & 4.5 & 3.7 & 3.1 & 2.4 & 1.9 & 1.5 \\
DAO 0 PSI & 4.2 & 3.3 & 2.6 & 1.8 & 1.2 & 0.7 & 0.1 \\
DAO 40 PSI & 2.6 & 1.8 & 0.9 & 0.4 & 0 & -0.5 & -1.1 \\
DAO 50 PSI & 2.6 & 1.8 & 1.1 & 0.4 & 0 & -0.7 & -1.8 \\
DAO 60 PSI & 2.6 & 1.6 & 0.8 & 0.3 & -0.3 & -0.9 & -1.9 \\
DAO 70 PSI & 2.6 & 1.6 & 0.7 & 0 & -0.5 & -1.2 & -2.3 \\
DAO 80 PSI & 1.8 & 0.8 & 0 & -0.7 & -1.4 & -2.2 & -4.2 \\
\hline
\end{tabular}

\section{Discussion}

\section{Offloading capacity of DAO}

The amount of force that the DAO relieved expressed as a percentage of bodyweight $\mathrm{BW}$, ranged from $11.3 \%$ at $40 \mathrm{PSI}$ to $30.5 \%$ at 80 PSI. It is currently unknown what amount of force relief at the ankle is clinically relevant, but through conversation with two orthotists, two general goals were set for the DAO. First, the DAO should provide axial unloading without compromising circulation or soft tissue integrity. The medical literature shows that during noninvasive ankle distraction no nerve damage was seen when tested up to $135 \mathrm{~N}$ for 1 hour. ${ }^{10}$ This is not directly applicable as this experiment was performed on an unloaded ankle (i.e., non-weight bearing) and the ankle would be under load with the DAO, but it still gave a reference of what was safe without causing nerve damage in an unloaded ankle. Second, a goal of at least $10 \%$ of bodyweight of force relief at the braced ankle was set as a mark of potential clinical efficacy. This test shows the ability of the DAO to provide offloading of the ankle. The DAO provided up to $148 \mathrm{~N}$ of brace force $\mathrm{Fb}$ to the user which amounted to $30.5 \%$ of body weight. The DAO was also able to transfer cylinder force output to brace force $\mathrm{Fb}$ at an $83-95 \%$ effectiveness rate. The DAO, therefore, accomplished both design goals set by the Orthotists.

\section{Mobility}

The DAO allowed for full functional ROM of ankle of 10 degrees of dorsiflexion and 20 degrees of plantar flexion. ${ }^{11}$ The Biodex testing showed that there was a reduction in resistive torque at the ankle with increasing DAO cylinder pressure. The measured passive moment values for the baseline native ankle condition are supported by literature. ${ }^{12}$ Normality of the data was confirmed using a ShapiroWilk Normality Test ( $\mathrm{p}=0.469)$ and an Equal Variance Test $(\mathrm{p}=0.953)$. A One-Way ANOVA showed that there is a difference between the mean resistive ankle moments of the bracing conditions $(\mathrm{p}=0.001)$ with a power of 0.926 . A Holm-Sidak Post- Hoc Analysis showed that the mean ankle moment for all DAO bracing conditions except "DAO OPSI" were significantly different from "Native Ankle." In other words, there was a reduction in baseline resistive moment at the ankle with increasing cylinder pressure, but no difference in the resistive moment of the ankle with the deactivated brace worn. Across the simulated range of motion (10 degrees dorsiflexion to 20 degrees plantar flexion), the native ankle experienced between 1.5 and $6.0 \mathrm{Nm}$ (4.5 Nm difference) of resistive moment. With the DAO donned and cylinders depressurized, between 4.2 and $0.1 \mathrm{Nm}$ (4.1 Nm moment difference) of resistive moment was measured during the motion. With the DAO donned and inflated to 50PSI, between 2.6 and $-1.8 \mathrm{Nm}$ (4.4Nm moment difference) of resistive moment was measured during the motion. With the DAO donned and inflated to 80 PSI, between 1.8 and $-4.2 \mathrm{Nm}$ (6Nm moment difference) of resistive moment was measured during the motion. The increased moment at 20 degrees plantar flexion was likely due to the ball joints of DAO hitting their limit (i.e., the ball joints were constructed to permit a maximum of 20 degrees of motion). In all pressurized bracing conditions, the mean resistive moment was reduced compared to the native ankle. This finding suggests that less torque was required to move the ankle when the activated brace was worn. There are two possible explanations for this. First, this could be because the natural compressive loads of the ankle are reduced by the distractive force of the brace, which reduces the congruency of the joint and in-turn allows for the joint surfaces to rotate and translate on each other more freely. Alternatively, this shift in values towards the negative could have been due to the brace force vector creating off-axis loads relative to the point of rotation of the Biodex footplate. In other words, the DAO may have been applying an external dorsiflexor moment due to alignment of the cylinders compared to the Biodex rotational axis, which manifested as a shift in the measured moment values towards the negative. Nevertheless, the resistive moment difference taken at the two extreme ends of motion for each condition was not significantly affected by the brace wear in a clinically relevant manner. Sagittal plane muscle moments for the ankle during gait were quantified by Sadeghi et al. ${ }^{13}$ The peak ankle moment measured during gait was a plantarflexor moment of $1.5 \mathrm{Nm} /$ $\mathrm{kg} \pm 1.20 \mathrm{Nm} / \mathrm{kg}$ at $48 \%$ of the gait cycle. For the individual tested in the present study, the maximum gait ankle moment could be estimated to be approximately $75 \mathrm{Nm}(50 \mathrm{~kg} \mathrm{x} 1.5 \mathrm{Nm} / \mathrm{kg})$. The greatest moment differences were found in the 70PSI and 80PSI bracing conditions, where the moment difference was $5.2 \mathrm{Nm}$ and $6 \mathrm{Nm}$, respectively. Compared to the native ankle at $4.5 \mathrm{Nm}$ moment difference, the $1.5 \mathrm{Nm}$ increase in resistive ankle moment only represents $2 \%$ of the maximal moment experienced by the ankle during gait, and it can be concluded that the presence of the DAO does not introduce additional resistance to natural ankle motion.

\section{Conclusion}

Benchtop validation of the DAO provided evidence of the efficacy of the DAO to provide clinically relevant offloading to the user while maintaining a functional range of motion without increasing the effort it takes to move the ankle. The next phase of device evaluation is to assess the functionality of the DAO in a clinical population with osteoarthritis of the ankle.

\section{Acknowledgements}

None.

\section{Conflict interest}

The author declares that they have no competing interests.

\section{References}

1. US. Department of Labor Bureau of Labor Statistics. News Release: Nonfatal Occupational Injuries and Illnesses Requiring Days Away from Work, 2014. November 19, 2015. 
2. Washington State Department of Labor \& Industries L\&I Workers' Compensation Claims 2007-2016 Number of Claims by Body Part Injured.

3. Barg A, Pagenstert GI, Hugle T, et al. Ankle osteoarthritis: etiology, diagnostics, and classification. Foot Ankle Clin. 2013;18(3):411-426.

4. Nguyen MP, Pedersen DR, Gao Y, et al. Intermediate-term follow- up after ankle distraction for treatment of end-stage osteoarthritis. J Bone Joint Surg Am. 2015;97(7):590-596.

5. Landro L. The Informed Patient: More Patients Opt to Replace Ankle Joints Instead of Fusion. The Wall Street Journal. 2014.

6. Belatti DA, Phisitkul P. Economic burden of foot and ankle surgery in the US Medicare population. Foot Ankle Int. 2014;35(4):334-40.

7. Kobayashi T, Leung AKL, Hutchins SW. Techniques to measure rigidity of ankle-foot orthosis: A review. J Rehabil Res Dev. 2011;48(5):565-576.

8. Kitaoka HB, Crevoisier XM, Harbst K, et al. The effect of custom-made braces for the ankle and hindfoot on ankle and foot kinematics and ground reaction forces. Arch Phys Med Rehabil. 2006;87(1):130-5.
9. DiDomenico LA, Gatalyak N. End-stage ankle arthritis: arthrodiastasis, supramalleolar osteotomy, or arthrodesis? Clin Podiatr Med Surg. 2012;29(3):391-412.

10. Dowdy PA, Watson BV, Amendola A, et al. Noninvasive ankle distraction: relationship between force, magnitude of distraction, and nerve conduction abnormalities. Arthroscopy. 1996;12(1):64-9.

11. Castro M. Ankle biomechanics. Foot and Ankle Clin N Am. 2002;7:67993.

12. Kay AD, Blazevich AJ. Moderate-duration static stretch reduces active and passive plantar flexor torque but not Achilles tendon stiffness or active muscle length. J Appl Physiol. 2009;106(4):1249-1256.

13. Sadeghi H, Sadeghi S, Prince F, et al. Functional roles of ankle and hip sagittal muscle moments in able-bodied gait. Clin Biomech (Bristol, Avon). 2001;16(8):688-95 